OCU-PHYS-420

AP-GR-120

\title{
A pair of extremal charged black holes on Kerr-Taub-bolt space
}

\author{
${ }^{1}$ Ken Matsuno*, ${ }^{1}$ Hideki Ishihara ${ }^{\dagger}$, and ${ }^{2}$ Masashi Kimura ${ }^{\ddagger}$ \\ ${ }^{1}$ Department of Mathematics and Physics, \\ Osaka City University, Sumiyoshi, Osaka 558-8585, Japan \\ ${ }^{2}$ DAMTP, University of Cambridge, \\ Centre for Mathematical Sciences, Wilberforce Road, \\ Cambridge CB3 OWA, United Kingdom
}

(Dated: September 3, 2018)

\begin{abstract}
We construct asymptotically Kaluza-Klein solutions in five-dimensional Einstein-Maxwell theory which represent a pair of extremal, charged, static black holes on Kerr-Taub-bolt space. Regularity conditions require that the topology of spatial infinity and that of each black hole are not $\mathrm{S}^{3}$, but different lens spaces. We show that for a given topology at spatial infinity, there are an infinite number of different horizon topologies for the black hole pair. We briefly discuss a generalization to the case with a positive cosmological constant.

PACS numbers: 04.50.-h, 04.70.Bw
\end{abstract}

\footnotetext{
* E-mail: matsuno@sci.osaka-cu.ac.jp

$\dagger$ E-mail: ishihara@sci.osaka-cu.ac.jp

$\ddagger$ E-mail: m.kimura@damtp.cam.ac.uk
} 


\section{INTRODUCTION}

In the context of string theories and brane world models, higher-dimensional spacetimes are actively discussed. Since the effects of extra dimensions would appear clearly in strong gravity regimes, we focus on higher-dimensional black hole spacetimes as a first step to reveal the higher-dimensional effects. If higher-dimensional black hole solutions have compactified extra dimensions, we can regard such black hole solutions as candidates of realistic models, since our observable world is effectively four-dimensional. We call these Kaluza-Klein black holes.

Comparing to spacetimes which consist of direct products of four-dimensional spacetimes and a compact extra dimension, spacetimes with a twisted compact extra dimension could admit a large isometry group. In such cases, we can obtain exact Kaluza-Klein black hole solutions easily. A family of five-dimensional squashed Kaluza-Klein black hole solutions [1 14] asymptote to effective four-dimensional spacetimes with a twisted $S^{1}$ as an extra dimension at infinity, and represent fully five-dimensional black holes near the squashed $\mathrm{S}^{3}$ horizons (See [15] as a review).

In this paper, we focus on Kaluza-Klein black holes in the five-dimensional spacetimes with a twisted compact extra dimension. In such spacetimes, even if we fix the spatial topology of infinity, and impose asymptotically locally flatness, there are various solutions of five-dimensional Kaluza-Klein black hole. We obtain new exact solutions that represent a pair of Kaluza-Klein black holes, and study such the possibility explicitly.

Because of nonlinearity of gravity, it is difficult to construct exact solutions that describe multi configuration of black holes. However, if we focus on the extremal charged black holes on Ricci-flat base spaces, Einstein-Maxwell equations reduce to Laplace's equations. In four-dimensional Einstein-Maxwell theory, due to the balance of gravitational attraction and electrical repulsion, we can obtain exact solutions that represent extremal charged multi-black holes [16, 17] by the superpositions of harmonic functions. Similarly, in fivedimensional Einstein-Maxwell theory, exact solutions of extremal charged multi-black holes are constructed in asymptotically flat spacetimes with the spatial topology of the threedimensional sphere [18, 19], in asymptotically locally flat spacetimes with the topology of the lens space [20 22], and in asymptotically Kaluza-Klein spacetimes [3], 6, 7, 11, 18, 23]. These five-dimensional multi-black hole solutions are constructed on four-dimensional Ricci- 
flat base spaces with different asymptotic structures.

In the five-dimensional multi-black hole spacetimes, the topology of each black hole is related to the topology of spatial infinity. For example, in the multi-black hole solutions on flat base space [18], both the topology of each black hole and that of spatial infinity are $\mathrm{S}^{3}$. On the other hand, in the multi-black hole solutions on Gibbons-Hawking base spaces [3, 6, 7, 11, 20 22], the topology of the $i$-th black hole is $S^{3}$ or the lens space $L\left(p_{i} ; 1\right)$ (natural numbers $p_{i} \neq 1$ ), and the topology of spatial infinity becomes the lens space $L\left(\sum_{i} p_{i} ; 1\right)$. In the present paper, we find new exact solutions where the rich variety of black hole topologies is allowed even if the topology of the spatial infinity is fixed.

We construct exact solutions in five-dimensional Einstein-Maxwell theory that describe a pair of extremal, charged, static black holes on Kerr-Taub-bolt base space [24], which is a generalization of the base spaces used in Refs. [3, 6 86, 11, 13, 20 22, 25, 26]. As discussed in Refs. [27, 28], Kerr-Taub-bolt space has conical singularities at the poles on the bolt. By using harmonic functions on Kerr-Taub-bolt space, we can put black holes on these points so that the conical singularities are concealed behind the horizons. Therefore, we can obtain five-dimensional black hole spacetimes that are regular on and outside the horizons. Inspecting the regularity, we find that the topology of each black hole and the topology of spatial infinity are not $S^{3}$ but lens space.

Kunduri and Lucietti found the black lens, black hole solutions with lens space topologies, with asymptotically globally flatness [29] by introducing solitonic disk objects and Chern-Simons term. On the other hand, we obtain regular multi-black lens solutions where asymptotic spatial topology is a different lens space for pure Einstein-Maxwell system. The multi-black hole solutions on Gibbons-Hawking space are known as the black lens solutions in asymptotic lens space topology [3, 6, 7, 11, 20 22]. These solutions admit only finite numbers of possibility for the topologies of black lens if the topology of infinity is fixed. In contrast, the present new solutions allow infinite sequences of lens space topologies of black holes. This shows that there exist rich possible topologies of Kaluza-Klein black hole spacetimes even if the topology of spatial infinity is fixed.

This paper is organized as follows. We present explicit forms of solutions in section II] Some known multi-black hole solutions are obtained by taking limits in our solutions. We show analytic extensions across the black hole horizons in section III. In section IV, we discuss regularity conditions and topology of solutions with an explicit example. We 
investigate possible horizon topologies under fixed topology of infinity in section $\mathrm{V}$. We give summary and discussion, and generalize our solutions to the solutions with a positive cosmological constant in section VI.

\section{SOLUTIONS}

We construct a pair of extremal, charged, static black holes on Kerr-Taub-bolt space as exact solutions in five-dimensional Einstein-Maxwell theory with the action

$$
S=\frac{1}{16 \pi} \int d^{5} x \sqrt{-g}\left(R-F_{\mu \nu} F^{\mu \nu}\right)
$$

The metric and the Maxwell field are given by

$$
\begin{aligned}
d s^{2} & =-H(r, \theta)^{-2} d t^{2}+H(r, \theta) d s_{4}^{2}, \\
A_{\mu} d x^{\mu} & = \pm \frac{\sqrt{3}}{2} H(r, \theta)^{-1} d t
\end{aligned}
$$

with

$$
\begin{aligned}
d s_{4}^{2}= & \Xi(r, \theta)\left[\frac{d r^{2}}{\Delta(r)}+d \theta^{2}\right]+\frac{\sin ^{2} \theta}{\Xi(r, \theta)}\left[2 \alpha \nu d \psi-\left(r^{2}-\nu^{2}-\alpha^{2}\right) d \phi\right]^{2} \\
& +\frac{\Delta(r)}{\Xi(r, \theta)}\left[2 \nu d \psi+\left(2 \nu \cos \theta+\alpha \sin ^{2} \theta\right) d \phi\right]^{2}, \\
\Delta(r)= & r^{2}-2 \mu r+\nu^{2}-\alpha^{2}, \quad \Xi(r, \theta)=r^{2}-(\nu-\alpha \cos \theta)^{2},
\end{aligned}
$$

and

$$
H(r, \theta)=1+\frac{m_{+}}{r-\mu-\left(r_{b}-\mu\right) \cos \theta}+\frac{m_{-}}{r-\mu+\left(r_{b}-\mu\right) \cos \theta},
$$

where $m_{ \pm}, \alpha, \mu, \nu, r_{b}:=\mu+\sqrt{\mu^{2}-\nu^{2}+\alpha^{2}}$ are constants. Equation (44) is the metric of four-dimensional Euclidean Kerr-Taub-bolt space [24], which is Ricci flat. ${ }^{1}$ Kerr-Taub-bolt space has a bolt at $r=r_{b}$, where $\Delta\left(r=r_{b}\right)=0 .^{2}$ If we require that the metric and the Maxwell field take the forms of (2) and (3), and the four-dimensional space $d s_{4}^{2}$ is Ricci flat, Einstein-Maxwell equations reduce to Laplace's equations. The function $H(r, \theta)$ in the form

\footnotetext{
1 Appearance of conical singularities in four-dimensional Kerr-Taub-bolt space is discussed in Refs. [27, 28].

2 A set of fixed points of a spatial Killing vector field, now it is $\partial / \partial \psi+2 \alpha \nu /\left(r_{b}^{2}-\nu^{2}-\alpha^{2}\right) \partial / \partial \phi$, is called a bolt if the set is two-dimensional manifold.
} 
of (6) is a harmonic function on Kerr-Taub-bolt space (4).$^{3}$ Two black holes are located at the north pole $(\theta=0)$ and the south pole $(\theta=\pi)$ on the bolt.

The coordinates run the ranges of $-\infty<t<\infty, r_{b}<r<\infty$, and $0 \leq \theta \leq \pi$. The ranges of $\phi$ and $\psi$ will be determined from the regularity conditions of the spacetime in section [V] For the spacetime signature to be $(-,+,+,+,+)$, the inequalities $r_{b}>0$ and $\Xi(r, \theta)>0$ should hold. Then we have the inequalities $\mu>0$ and $\mu>|\nu|$. Since we can consider the case $\alpha>0$ and $\nu>0$ by rearrangement of the angular coordinates without loss of generality, in this paper, we restrict ourselves to the ranges of parameters such that ${ }^{4}$

$$
m_{ \pm}>0, \quad \alpha>0, \quad \mu>\nu>0
$$

\section{A. Limits}

By taking some limits in the solution (2), we obtain some known, extremal, charged, static, asymptotically locally flat, regular black hole solutions. First, when $\alpha=0, \mu=\nu$, the bolt shrinks to a NUT singularity, and the solution coincides with the single black hole solution on self-dual Taub-NUT base space [3, 5]. Secondly, when $\alpha=0, \mu=\nu\left[1+a^{4} /\left(128 \nu^{4}\right)\right]$, then taking the limit $\nu \rightarrow \infty$ with $\tilde{r}^{2}=4\left(r^{2}-\nu^{2}\right)$ held fixed, the solution represents a pair of black holes on Eguchi-Hanson base space [20]. Thirdly, when $\alpha=\nu=0$, the solution represents a pair of black holes on a Kaluza-Klein bubble (equivalently, on Euclidean Schwarzschild base space) [25, 26]. Fourthly, when $\alpha=0$, the solution represents a pair of black holes on Taub-bolt base space [13]. Lastly, when $\nu=0$, the solution represents a pair of black holes on Euclidean Kerr base space [8].

3 The harmonic function (6) is a generalization of the harmonic functions on Taub-bolt space [9] and Eguchi-Hanson space [20].

4 The solution (2) is not a supersymmetric solution since Kerr-Taub-bolt base space (4) is not a hyperKähler space in the ranges of parameters (7). 


\section{B. Asymptotic behavior}

The asymptotic behavior of the metric (2) near the infinity $r \rightarrow \infty$ becomes

$$
\begin{aligned}
d s^{2} \simeq & -\left(1-2 \frac{m_{+}+m_{-}}{r}\right) d t^{2}+\left(1+\frac{m_{+}+m_{-}+2 \mu}{r}\right) d r^{2} \\
& +r^{2}\left(1+\frac{m_{+}+m_{-}}{r}\right)\left(d \theta^{2}+\sin ^{2} \theta d \phi^{2}\right) \\
& +4 \nu^{2}\left(1+\frac{m_{+}+m_{-}-2 \mu}{r}\right)(d \psi+\cos \theta d \phi)^{2} .
\end{aligned}
$$

We see that this solution is asymptotically locally flat, i.e., the metric asymptotes to a twisted constant $\mathrm{S}^{1}$ fiber bundle over the four-dimensional Minkowski spacetime, and the parameter $\nu$ denotes the size of extra dimension. However, we cannot take the limit $\nu \rightarrow \infty$ while keeping the radius of the bolt finite. This is because $r_{b}=\mu+\sqrt{\mu^{2}-\nu^{2}+\alpha^{2}}$ is larger than $\nu$ if $\mu>\nu>0$.

As is seen later, we need to require that the topology of $r=$ const. $\left(>r_{b}\right)$ surface is a lens space $L(p ; q)$ for coprime natural numbers $p$ and $q$ with $p>q>0$ (see Appendix $\mathrm{A}$ for the definition of a lens space). The Komar mass $M$ and the total electric charge $Q$ at the infinity are given by

$$
M=\frac{\sqrt{3}}{2}|Q|=\frac{3 \nu}{\pi}\left(m_{+}+m_{-}\right) \frac{\mathcal{A}_{\mathrm{S}^{3}}}{p},
$$

where $\mathcal{A}_{\mathrm{S}^{3}}=2 \pi^{2}$ denotes the area of a unit $\mathrm{S}^{3}$.

\section{ANALYTIC EXTENSIONS ACROSS THE BLACK HOLE HORIZONS}

In this section, we show that two black holes are located at the north pole $(\theta=0)$ and

the south pole $(\theta=\pi)$ on the bolt $\left(r=r_{b}\right)$. In the coordinate system $(t, r, \theta, \phi, \psi)$, the metric (2) with (44)-(17) diverges apparently at $r=r_{b}, \theta=0$ and $r=r_{b}, \theta=\pi$.

In order to remove these coordinate singularities we introduce new coordinates 
$\left(v_{ \pm}, \rho_{ \pm}, \Theta_{ \pm}, \Phi_{ \pm}, \Psi_{ \pm}\right)$such that

$$
\begin{aligned}
& d v_{ \pm}-F_{ \pm}\left(\rho_{ \pm}, \Theta_{ \pm}\right) d \rho_{ \pm} \\
& -d \Theta_{ \pm}\left(\sqrt{\frac{m_{ \pm}^{3}\left(r_{b} \mu+\nu(-\nu \pm \alpha)\right)}{\left(r_{b}-\mu\right)^{3}}} \sin \Theta_{ \pm} \cos ^{3} \Theta_{ \pm}\right. \\
& \left.\quad+\int d \rho_{ \pm} \partial_{\Theta_{ \pm}} F_{ \pm}\left(\rho_{ \pm}, \Theta_{ \pm}\right)\right)=d t \\
& 4\left(r_{b}-\mu\right) \rho_{ \pm} \cos ^{2} \Theta_{ \pm}=r-r_{b}, \\
& 8 \rho_{ \pm} \sin ^{2} \Theta_{ \pm}=\left(\theta-\frac{\pi}{2} \pm \frac{\pi}{2}\right)^{2} \\
& \Phi_{ \pm}= \pm \frac{r_{b}^{2}-\nu^{2}-\alpha^{2}}{r_{b}^{2}-(\nu \mp \alpha)^{2}}\left(\frac{2 \alpha \nu}{r_{b}^{2}-\nu^{2}-\alpha^{2}} \psi-\phi\right), \\
& \Psi_{ \pm}=\frac{2 \nu\left(r_{b}-\mu\right)}{r_{b}^{2}-(\nu \mp \alpha)^{2}}(\psi \pm \phi),
\end{aligned}
$$

where the functions $F_{ \pm}\left(\rho_{ \pm}, \Theta_{ \pm}\right)$are defined by

$$
F_{ \pm}^{2}\left(\rho_{ \pm}, \Theta_{ \pm}\right)=\frac{2 H^{3} \Xi}{\Delta \rho_{ \pm}}\left(\Delta \sin ^{2} \Theta_{ \pm}+8 \rho_{ \pm}\left(r_{b}-\mu\right)^{2} \cos ^{4} \Theta_{ \pm}\right)
$$

and $0 \leq \Theta_{ \pm} \leq \pi / 2$. We show the relation between the coordinates $(r, \theta)$ and $\left(\rho_{ \pm}, \Theta_{ \pm}\right)$in Fig 1.

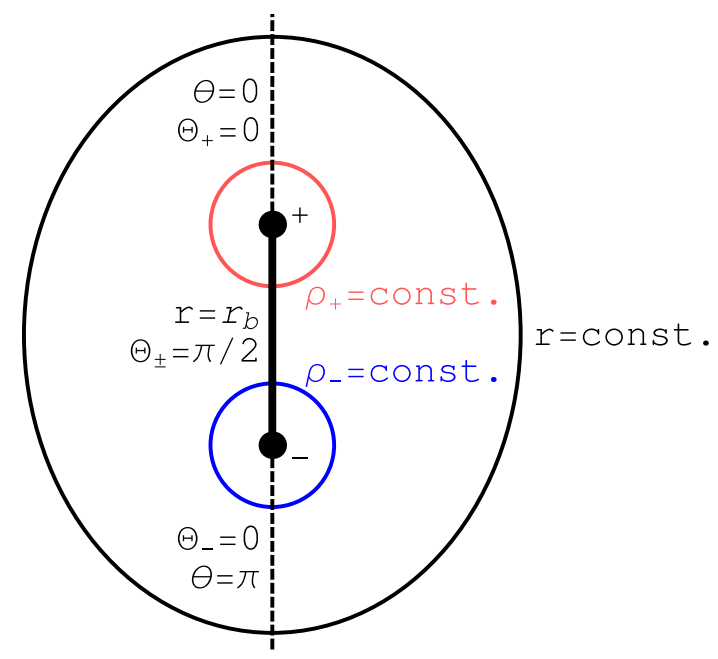

FIG. 1: The relation between the coordinates $(r, \theta)$ and $\left(\rho_{ \pm}, \Theta_{ \pm}\right)$. The bolt $r=r_{b}$ is shown by the thick segment. The northern black hole on the bolt is denoted by the dot ' + ' and the southern one by the dot '-'. The symmetric polar axes $\theta=0$ and $\theta=\pi$ are denoted by the broken lines, respectively. 
In these coordinates, the metric (2) behaves as

$$
\begin{aligned}
d s^{2} \simeq 8 & \sqrt{\frac{\left(r_{b}-\mu\right)\left(r_{b} \mu+\nu(-\nu \pm \alpha)\right)}{m_{ \pm}}} d v_{ \pm} d \rho_{ \pm} \\
& +\frac{4 m_{ \pm}\left(r_{b} \mu+\nu(-\nu \pm \alpha)\right)}{r_{b}-\mu}\left(d \Theta_{ \pm}^{2}+\sin ^{2} \Theta_{ \pm} d \Phi_{ \pm}^{2}+\cos ^{2} \Theta_{ \pm} d \Psi_{ \pm}^{2}\right)+O\left(\rho_{ \pm}\right),
\end{aligned}
$$

near $\rho_{ \pm}=0$. The metric well behaves at the null surfaces $\rho_{ \pm}=0$. We see that $\eta=\partial / \partial t=$ $\partial / \partial v_{ \pm}$is a Killing vector field that becomes null at $\rho_{ \pm}=0$, and $\eta$ is hypersurface orthogonal to the surfaces $\rho_{ \pm}=0$. These mean that the null hypersurfaces $\rho_{ \pm}=0$ are Killing horizons. From the next-order terms of the metric, we see the norm of the Killing vector field $\partial / \partial v_{ \pm}$ is proportional to $\rho_{ \pm}^{2}$ near $\rho_{ \pm}=0$. Then the surface gravity vanishes, and the solution is extremal. Hence the solution (2) with (4)-(7) describes a pair of extremal charged black holes on Kerr-Taub-bolt base space.

In fact, one can easily check that the set of new coordinates $\left(v_{ \pm}, \rho_{ \pm}, \Theta_{ \pm}, \Phi_{ \pm}, \Psi_{ \pm}\right)$gives analytic extensions across the horizons. So the spacetime has no curvature singularity on and outside the horizons.

\section{REGULARITY CONDITIONS AND TOPOLOGY}

In this section we discuss the regularity conditions for the absence of conical singularities on the spacetime. The metrics of angular parts of the black hole horizons in equation (16) are locally round $\mathrm{S}^{3}$. In order to remove the conical singularities at $\Theta_{ \pm}=0, \pi / 2$, we should impose the periodic conditions

$$
\left(\Phi_{ \pm}, \Psi_{ \pm}\right) \sim\left(\Phi_{ \pm}+2 \pi, \Psi_{ \pm}\right)
$$

at $\Theta_{ \pm}=0$, and

$$
\left(\Phi_{ \pm}, \Psi_{ \pm}\right) \sim\left(\Phi_{ \pm}, \Psi_{ \pm}+2 \pi\right)
$$

at $\Theta_{ \pm}=\pi / 2$.

We translate the conditions for $\left(\Phi_{ \pm}, \Psi_{ \pm}\right)$to a coordinate system which covers the regions around the north pole and the south pole simultaneously. Via the original coordinate system $(\phi, \psi)$, we introduce such the coordinates as

$$
\Phi=\frac{1}{2}(\psi-\phi), \quad \Psi=\frac{1}{2}(\psi+\phi) .
$$


Using the relations (13), (14), and (19) we have

$$
\begin{array}{ll}
\Phi_{+}=\Phi-\frac{\sigma_{1}}{\sigma_{2}} \Psi, & \Psi_{+}=\frac{1}{\sigma_{2}} \Psi, \\
\Phi_{-}=\Psi-\frac{\sigma_{2}}{\sigma_{1}} \Phi, & \Psi_{-}=\frac{1}{\sigma_{1}} \Phi,
\end{array}
$$

where $\sigma_{1}$ and $\sigma_{2}$ are defined by

$$
\sigma_{1}=\frac{r_{b}^{2}-(\nu+\alpha)^{2}}{4 \nu\left(r_{b}-\mu\right)}, \quad \sigma_{2}=\frac{r_{b}^{2}-(\nu-\alpha)^{2}}{4 \nu\left(r_{b}-\mu\right)} .
$$

We note that $\sigma_{1}>0$ and $\sigma_{2}>0$ in the ranges of parameters (7). The four conditions (17) and (18) reduce to the three conditions

$$
\begin{aligned}
& (\Phi, \Psi) \sim(\Phi, \Psi+2 \pi), \\
& (\Phi, \Psi) \sim(\Phi+2 \pi, \Psi),
\end{aligned}
$$

and

$$
(\Phi, \Psi) \sim\left(\Phi+2 \pi \sigma_{1}, \Psi+2 \pi \sigma_{2}\right) .
$$

The regularity conditions (17) at the north pole axis of the northern black hole $\left(\Theta_{+}=0\right)$, and at the north pole axis of the southern black hole $\left(\Theta_{-}=0\right)$ correspond to the regularity conditions (23) and (24) at the north pole axis $(\theta=0)$ and the south pole axis $(\theta=\pi)$ of the $r=$ const. $\left(>r_{b}\right)$ surface. On the other hand, the regularity conditions (18) at the south pole axes of two black holes $\left(\Theta_{ \pm}=\pi / 2\right)$ reduce to one condition (25) which corresponds to the regularity condition on the bolt off the horizons (see Fig,1 and Appendix B).

From the inequalities (7) for $\alpha, \mu$, and $\nu$ we see that

$$
0<\sigma_{2}-\sigma_{1}<1<\sigma_{1}+\sigma_{2} .
$$

If $\sigma_{1}$ and $\sigma_{2}$ were 0 or integers which are coprime, the metric (2) with the regularity conditions (23) and (24) would mean that the $r=$ const. $\left(>r_{b}\right)$ surface is topologically $S^{3}$. However, the inequality (26) does not permit the case. The condition (25) with (26) requires that the point $(\Phi, \Psi)=(0,0)$ should be identified with a point in the region $\Phi<\Psi<\Phi+2 \pi$, and $2 \pi<\Phi+\Psi$. Namely, the condition (25) is an additional identification, then we should consider the case that the $r=$ const. $\left(>r_{b}\right)$ surface is a lens space $L(p ; q)$, which is specified by the additional identification condition

$$
(\Phi, \Psi) \sim\left(\Phi+2 \pi \frac{q}{p}, \Psi+2 \pi \frac{1}{p}\right),
$$


where $p, q$ are natural numbers which are coprime. The identification (27) in the present paper is graphically shown in Appendix A. We should note that the lens spaces $L(p ; q)$ and $L(p ; p-q)$ are topologically equivalent, however, these spaces with the metric (2) are different in geometry.

We show all the conditions (23), (24), (25), and (27) are compatible if the parameters $\alpha$ and $\mu$ are chosen suitably. In order that the conditions (25) and (27) are compatible, $\sigma_{1}$ and $\sigma_{2}$ are given by

$$
\sigma_{1}=\frac{k_{1}}{p}, \quad \sigma_{2}=\frac{k_{2}}{p}
$$

where $k_{1}, k_{2}$, and $p$ are natural numbers which are coprime to each other. If these are not coprime, there appears a shorter period of identification so that a conical singularity emerges. The inequality (26) means

$$
0<k_{2}-k_{1}<p<k_{1}+k_{2}
$$

Since $k_{2}$ and $p$ are coprime, there exists a natural number $n$ such that $\left(n k_{2} \bmod p\right)=1 .^{5}$ By the use of the number $n$, the parameter $q$ is given by

$$
q=\left(n k_{1} \bmod p\right)
$$

It is easy to see that $p$ and $q$ are coprime.

Rewriting the conditions (23), (24), and (25) in the coordinates $\left(\Phi_{ \pm}, \Psi_{ \pm}\right)$we have the conditions (17), (18), and

$$
\begin{aligned}
& \left(\Phi_{+}, \Psi_{+}\right) \sim\left(\Phi_{+}-2 \pi \frac{k_{1}}{k_{2}}, \Psi_{+}+2 \pi \frac{p}{k_{2}}\right), \\
& \left(\Phi_{-}, \Psi_{-}\right) \sim\left(\Phi_{-}-2 \pi \frac{k_{2}}{k_{1}}, \Psi_{-}+2 \pi \frac{p}{k_{1}}\right) .
\end{aligned}
$$

If the conditions (31) and (32) were absent, the topologies of black holes would be $\mathrm{S}^{3}$, but in fact the existence of them in addition to (17) and (18) requires that the topologies of the northern and the southern black hole horizons are $L\left(p_{+} ; q_{+}\right)$and $L\left(p_{-} ; q_{-}\right)$, where $\left(p_{+}, q_{+}\right)$and $\left(p_{-}, q_{-}\right)$denote two sets of coprime natural numbers. Here, two points

5 For integers $a$ and $b(>0)$, we understand that the remainder of the division of $a$ by $b$ is denoted by $(a \bmod b)$, where $0 \leq(a \bmod b) \leq b-1$. 
$(\Phi, \Psi)=(q / p, 1 / p)$ and $(1 / p, \tilde{q} / p)$ directly determine the topological parameters, where $\tilde{q}$ is the natural number less than $p$ such that

$$
(q \tilde{q} \bmod p)=1
$$

Substituting $(\Phi, \Psi)=(q / p, 1 / p)$ into (20), and $(\Phi, \Psi)=(1 / p, \tilde{q} / p)$ into (21), we have

$$
\begin{aligned}
& \left(\Phi_{+}, \Psi_{+}\right)=\left(\frac{1}{k_{2}} \frac{1}{p}\left(q k_{2}-k_{1}\right), \frac{1}{k_{2}}\right)=\left(\frac{q_{+}}{p_{+}}, \frac{1}{p_{+}}\right), \\
& \left(\Phi_{-}, \Psi_{-}\right)=\left(\frac{1}{k_{1}} \frac{1}{p}\left(\tilde{q} k_{1}-k_{2}\right), \frac{1}{k_{1}}\right)=\left(\frac{q_{-}}{p_{-}}, \frac{1}{p_{-}}\right) .
\end{aligned}
$$

Then the lens parameters $p_{ \pm}$and $q_{ \pm}$are given by

$$
\begin{aligned}
& p_{+}=k_{2}, \quad q_{+}=\frac{1}{p}\left(q k_{2}-k_{1}\right), \\
& p_{-}=k_{1}, \quad q_{-}=\frac{1}{p}\left(\tilde{q} k_{1}-k_{2}\right) .
\end{aligned}
$$

From (29), (36), and (37), we see that the lens parameters of the northern and the southern black holes and spatial infinity, $p_{ \pm}$and $p$, are coprime to each other, and satisfy

$$
0<p_{+}-p_{-}<p<p_{+}+p_{-} .
$$

Solving (22) for $\alpha$ and $\mu$, we have

$$
\begin{aligned}
& \frac{\alpha}{\nu}=\frac{2 p}{k_{2}-k_{1}} \sqrt{\frac{k_{1} k_{2}}{p^{2}-\left(k_{2}-k_{1}\right)^{2}}-\frac{k_{1}+k_{2}}{k_{2}-k_{1}}}, \\
& \frac{\mu}{\nu}=\frac{p\left(k_{1}+k_{2}\right)-2 \sqrt{k_{1} k_{2}\left(p^{2}-\left(k_{2}-k_{1}\right)^{2}\right)}}{\left(k_{2}-k_{1}\right)^{2}} .
\end{aligned}
$$

The physical parameters $\alpha / \nu$ and $\mu / \nu$ take discrete values. Then $r_{b} / \nu$ also has a discrete value

$$
\frac{r_{b}}{\nu}=2 \sqrt{\frac{k_{1} k_{2}}{p^{2}-\left(k_{2}-k_{1}\right)^{2}}} .
$$

The set of three natural numbers $p, k_{1}$, and $k_{2}$ determines the lens parameters $q, p_{+}, q_{+}, p_{-}$, and $q_{-}$. That is, the topology of the $r=$ const. surface, $L(p ; q)$, the northern black hole horizon, $L\left(p_{+} ; q_{+}\right)$, and the southern black hole horizon, $L\left(p_{-} ; q_{-}\right)$, are specified by $p, k_{1}$, and $k_{2}$. The areas of the northern and the southern black holes with lens space topologies are given by

$$
\mathcal{A}_{ \pm}=\left[\frac{4 m_{ \pm}\left(r_{b} \mu+\nu(-\nu \pm \alpha)\right)}{r_{b}-\mu}\right]^{3 / 2} \frac{1}{p_{ \pm}} \mathcal{A}_{\mathrm{S}^{3}}
$$


respectively.

Since $p_{+}=k_{2}>1$ and $p_{-}=k_{1}>1$, the topologies of black holes cannot be $\mathrm{S}^{3}$ if $\alpha>0, \mu>\nu>0 .{ }^{6}$ This implies that conical singularities appear if we take no black hole limit $m_{ \pm} \rightarrow 0$. This result is consistent with the discussions in Refs. [27, 28]. Even though Euclidean Kerr-Taub-bolt space (4) has conical singularities at the north and the south poles on the bolt, we can obtain regular multi-black hole solution (2) with (4)-(17) by putting black holes on them. ${ }^{7}$

\section{A. Example: The case of given $p, k_{1}, k_{2}$}

Let us consider the case of $p=3, k_{1}=4, k_{2}=5$ as an example. We determine the lens parameters $p, q$, and $p_{ \pm}, q_{ \pm}$by (30), (36), and (37).

First, we see the lens parameter for the $r=$ const. $\left(>r_{b}\right)$ surface. We find $n=2$ satisfies

$$
\left(n k_{2} \bmod p\right)=(n \times 5 \bmod 3)=1 .
$$

Then, we obtain

$$
q=\left(n k_{1} \bmod p\right)=(2 \times 4 \bmod 3)=2
$$

The topology of the $r=$ const. $\left(>r_{b}\right)$ surface is $L(3 ; 2)$.

Next, we see the black hole horizons. We see that

$$
p_{+}=k_{2}=5, \quad q_{+}=\frac{1}{p}\left(q k_{2}-k_{1}\right)=\frac{1}{3}(2 \times 5-4)=2 .
$$

The topology of the northern black hole horizon is $L(5 ; 2)$.

Similarly, we find $\tilde{q}=2$ solves

$$
(q \tilde{q} \bmod p)=(2 \tilde{q} \bmod 3)=1
$$

Then, we have

$$
p_{-}=k_{1}=4, \quad q_{-}=\frac{1}{p}\left(\tilde{q} k_{1}-k_{2}\right)=\frac{1}{3}(2 \times 4-5)=1 .
$$

6 If we take the limit $\alpha \rightarrow 0$, the base space reduces to Euclidean Taub-bolt space which is regular everywhere. In this case, the topology of black hole can be $\mathrm{S}^{3}$.

7 This method is similar to the constructions of the multi-black hole solutions on Gibbons-Hawking space [3, 6, 7, 20-22]. 
The topology of the southern black hole horizon is $L(4 ; 1)$.

The same thing can be understood graphically. Three conditions (23), (24), and (25) for the case $p=3, k_{1}=4$, and $k_{2}=5$ are compatible if the $r=$ const. $\left(>r_{b}\right)$ surface is $L(3 ; 2)$ (see Fig 2 and Appendix (A). Transforming identification points in the $\Phi-\Psi$ plane into the $\Phi_{ \pm}-\Psi_{ \pm}$plane, we find the northern and the southern black hole horizons are $L(5 ; 2)$ and $L(4 ; 1)$ (see Fig, 3 ).

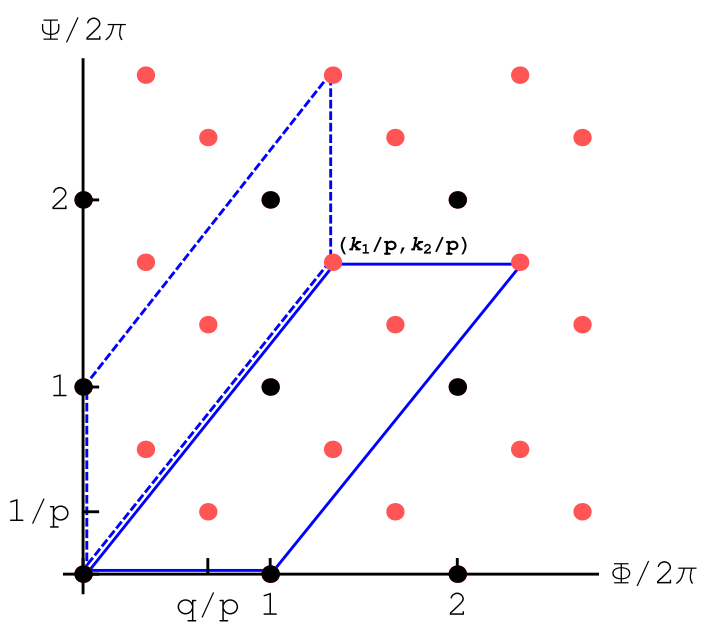

FIG. 2: The identifications in the case of $p=3, k_{1}=4, k_{2}=5$. All points which are identified with $(0,0)$ are plotted in the $\Phi / 2 \pi-\Psi / 2 \pi$ plane. The points described by (23) and (24) are shown by black dots, and the points for (25) are by dark (red) dots. We see that $q=2$ in this case, then the topology of the $r=$ const. $\left(>r_{b}\right)$ surface is $L(3 ; 2)$. The solid and dashed parallelograms correspond to the squares $((0,0),(1,0),(1,1),(0,1))$ in the $\Phi_{+} / 2 \pi-\Psi_{+} / 2 \pi$ and $\Phi_{-} / 2 \pi-\Psi_{-} / 2 \pi$ planes, respectively (see Fig鸟).

The parameters $\alpha, \mu$, and $r_{b}$ are obtained as

$$
\frac{\alpha}{\nu}=6 \sqrt{\frac{5}{2}}-9, \quad \frac{\mu}{\nu}=27-8 \sqrt{10}, \quad \text { and } \quad \frac{r_{b}}{\nu}=\sqrt{10} .
$$

\section{POSSIBLE HORIZON TOPOLOGY UNDER FIXED TOPOLOGY OF INFIN- ITY}

It is interesting that there are rich possibilities of lens space topologies of the northern and the southern black hole horizons even if the topology of spatial boundary at infinity, equivalently, the topology of $r=$ const. $\left(>r_{b}\right)$ surface, is fixed. Namely, for given $p$, there 

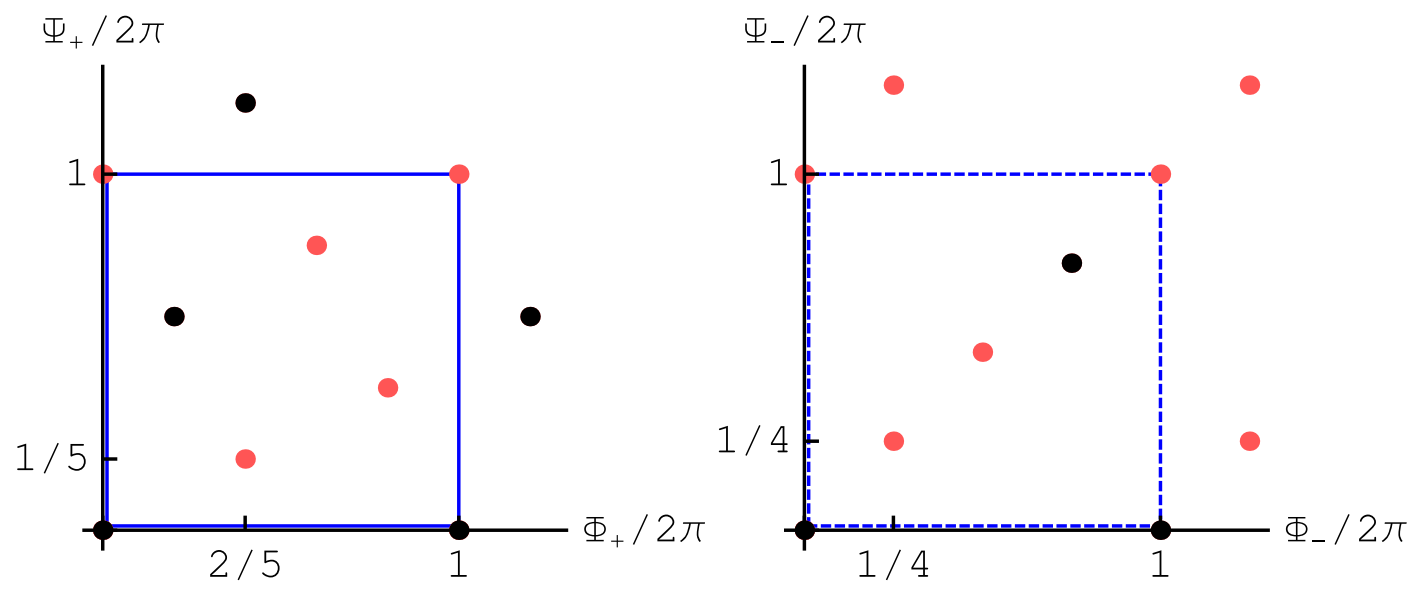

FIG. 3: The points which are identified with $(0,0)$ are plotted in the $\Phi_{+} / 2 \pi-\Psi_{+} / 2 \pi$ plane (the left panel). The solid square corresponds to the solid parallelogram in Fig.2. We see that the topology of the northern black hole horizon is $L(5 ; 2)$. The same is shown in the $\Phi_{-} / 2 \pi-\Psi_{-} / 2 \pi$ plane (the right panel). The dashed square corresponds to the dashed parallelogram in Fig,2. The topology of the southern black hole horizon is $L(4 ; 1)$.

are a variety of sets, $k_{1}$ and $k_{2}$, which give the same $q$.

If we fix the topology of spatial infinity as $L(p ; q)$, from (A4) we see that such the $k_{1}$ and $k_{2}$ are given by

$$
k_{1}=a q-b p, \quad k_{2}=a,
$$

where $a$ and $b$ are natural numbers such that $a, b$, and $p$ are coprime, so that $k_{1}, k_{2}$, and $p$ are coprime to each other. Similarly, paying attention to the point $(\Phi, \Psi)=(1 / p, \tilde{q} / p)$, we see that

$$
k_{1}=\tilde{a}, \quad k_{2}=\tilde{a} \tilde{q}-\tilde{b} p
$$

where $\tilde{a}$ and $\tilde{b}$ are natural numbers such that

$$
\tilde{a}=a q-b p, \quad a=\tilde{a} \tilde{q}-\tilde{b} p,
$$

and the definition of $\tilde{q}$ is given by (33).

Since the parameters $k_{1}$ and $k_{2}$ satisfy the inequality (29) then from (49) and (50) we have

$$
0<-a(q-1)+b p=\tilde{a}(\tilde{q}-1)-\tilde{b} p<p .
$$


Then, we can set

$$
-a(q-1)+b p=\tilde{a}(\tilde{q}-1)-\tilde{b} p=l,
$$

where $l$ is a natural number in the range $0<l<p$. Then, $a, b$ and $\tilde{a}, \tilde{b}$ satisfying (53) are labeled by $l$ as $a_{l}, b_{l}$ and $\tilde{a}_{l}, \tilde{b}_{l}$, respectively. Equation (53) means that $-b_{l}$ is the quotient of $-a_{l}(q-1)$ devided by $p$, and $l$ is the remainder. At the same time, $\tilde{b}_{l}$ is the quotient of $\tilde{a}_{l}(\tilde{q}-1)$ devided by $p$, and $l$ is the remainder. Then, $a_{l}$ and $\tilde{a}_{l}$ are expressed by

$$
\left(-a_{l}(q-1) \bmod p\right)=l, \quad\left(\tilde{a}_{l}(\tilde{q}-1) \bmod p\right)=l .
$$

For a given value of $l$, the parameter $a_{l}$ and $\tilde{a}_{l}$ are infinite sequences of natural numbers. The parameters $k_{1}$ and $k_{2}$ written as (49) and (50) are also written by $a_{l}, \tilde{a}_{l}$, and $l$ as

$$
k_{1}=\tilde{a}_{l}=a_{l}-l, \quad k_{2}=a_{l}=\tilde{a}_{l}+l .
$$

Here, $a_{l}$ and $\tilde{a}_{l}$ are restricted such that $k_{1}$ and $k_{2}$ are coprime. Furthermore, the inequality (29) requires

$$
p<2 a_{l}-l=2 \tilde{a}_{l}+l
$$

We see that there is no $a_{l}$ or $\tilde{a}_{l}$ for $q=1$, in other words, we find no $\left(k_{1}, k_{2}\right)$ in the region (29). Otherwise, the infinite sequences of $a_{l}, \tilde{a}_{l}$ give infinite sequences of $k_{1}, k_{2}$, therefore there exist infinite sequences of $p_{ \pm}$and $q_{ \pm}$. This means that the black holes can take infinite sequences of lens space topologies even if the topology of the spatial boundary at infinity is fixed.

Substituting (53) and (55) for $a_{l}, b_{l}$ and $\tilde{a}_{l}, \tilde{b}_{l}$ into (36), (37), we obtain ${ }^{8}$

$$
\begin{aligned}
& p_{+}=a_{l}, \quad q_{+}=b_{l}, \\
& p_{-}=\tilde{a}_{l}, \quad q_{-}=\tilde{b}_{l} .
\end{aligned}
$$

Substituting (55) into (39), (40), and (41), we see the geometrical parameters are written

8 Using $p, q, \tilde{q}, l, a_{l}$, we can write $p_{ \pm}$and $q_{ \pm}$as $p_{+}=a_{l}, q_{+}=\left(l+a_{l}(q-1)\right) / p, p_{-}=a_{l}-l, q_{-}=$ $\left(\left(a_{l}-l\right)(\tilde{q}-1)-l\right) / p$. 
as

$$
\begin{aligned}
\frac{\alpha}{\nu} & =\frac{2 p}{l} \sqrt{\frac{a_{l}\left(a_{l}-l\right)}{p^{2}-l^{2}}}-\frac{2 a_{l}-l}{l}, \\
\frac{\mu}{\nu} & =\frac{1}{l^{2}}\left(p\left(2 a_{l}-l\right)-2 \sqrt{a_{l}\left(a_{l}-l\right)\left(p^{2}-l^{2}\right)}\right), \\
\frac{r_{b}}{\nu} & =2 \sqrt{\frac{a_{l}\left(a_{l}-l\right)}{p^{2}-l^{2}}} .
\end{aligned}
$$

We see that the size of the bolt $r_{b}$ becomes large as $a_{l}$ increases. In this spacetime, the topologies of black holes are tightly related to the geometrical parameters.

\section{A. Example: The case of fixed $p, q$}

1. $p=4, q=3$ case

In this case, we obtain $\tilde{q}=3$. From (54) and (55), we obtain $a_{l}$ and $\tilde{a}_{l}$ for only $l=2$ as

$$
a_{2}=2 j+1=k_{2}, \quad \tilde{a}_{2}=2 j-1=k_{1}, \quad(j=2,3, \cdots),
$$

where $j$ is restricted to satisfy the inequalities (52) and (56) with (53) (see Fig 44). From (51), we have

$$
b_{2}=j+1, \quad \tilde{b}_{2}=j-1 .
$$

Then,

$$
p_{+}=2 j+1, \quad q_{+}=j+1 ; \quad p_{-}=2 j-1, \quad q_{-}=j-1, \quad(j=2,3, \cdots) .
$$

The geometrical parameters $\left(\alpha, \mu, r_{b}\right)$ are respectively given by

$$
\begin{aligned}
\frac{\alpha}{\nu} & =\frac{2 \sqrt{4 j^{2}-1}}{\sqrt{3}}-2 j, \\
\frac{\mu}{\nu} & =4 j-\sqrt{3\left(4 j^{2}-1\right)}, \\
\frac{r_{b}}{\nu} & =\frac{\sqrt{4 j^{2}-1}}{\sqrt{3}} .
\end{aligned}
$$


2. $p=5, q=2$ case

In this case, we find $\tilde{q}=3$ that satisfies (33). There are four possible sequences of $a_{l}=k_{2}, \quad \tilde{a}_{l}=k_{1},(l=1,2,3,4)$ (see Fig 5atisfying (54) as

$$
\begin{array}{lll}
l=1: & a_{1}=5 j-1, \quad \tilde{a}_{1}=5 j-2, & (j=1,2,3, \cdots), \\
l=2: & a_{2}=5 j+3, \quad \tilde{a}_{2}=5 j+1, \quad(j=2,4,6, \cdots), \\
l=3: & a_{3}=5 j+2, \quad \tilde{a}_{3}=5 j-1, \quad(j=1,3,4,6, \cdots,(j \bmod 3) \neq 2), \\
l=4: & a_{4}=5 j+6, \quad \tilde{a}_{4}=5 j+2, \quad(j=1,3,5, \cdots),
\end{array}
$$

where $j$ is restricted so that $a_{l}$ and $\tilde{a}_{l}$ are coprime, and the inequalities (52) and (56) with (53) are satisfied. From (51), we have

$$
\begin{array}{ll}
l=1: & b_{1}=j, \quad \tilde{b}_{1}=2 j-1, \\
l=2: & b_{2}=j+1, \quad \tilde{b}_{2}=2 j, \\
l=3: & b_{3}=j+1, \quad \tilde{b}_{3}=2 j-1, \\
l=4: & b_{4}=j+2, \quad \tilde{b}_{4}=2 j .
\end{array}
$$

Then, from (57), we determine $p_{ \pm}, q_{ \pm}$as follows

$$
\begin{gathered}
l=1: \quad p_{+}=5 j-1, \quad q_{+}=j ; \quad p_{-}=5 j-2, \quad q_{-}=2 j-1, \\
(j=1,2,3, \cdots), \\
l=2: \quad p_{+}=5 j+3, \quad q_{+}=j+1 ; \quad p_{-}=5 j+1, \quad q_{-}=2 j, \\
\quad(j=2,4,6 \cdots), \\
l=3: \quad p_{+}=5 j+2, \quad q_{+}=j+1 ; \quad p_{-}=5 j-1, \quad q_{-}=2 j-1, \\
\quad(j=1,3,4,6, \cdots,(j \bmod 3) \neq 2), \\
l=4: \quad p_{+}=5 j+6, \quad q_{+}=j+2 ; \quad p_{-}=5 j+2, \quad q_{-}=2 j, \\
\quad(j=1,3,5, \cdots) .
\end{gathered}
$$

\section{SUMMARY AND DISCUSSION}

We construct a pair of extremal, charged, static black holes on Euclidean Kerr-Taubbolt base space in five-dimensional Einstein-Maxwell theory. The metric asymptotes to an 


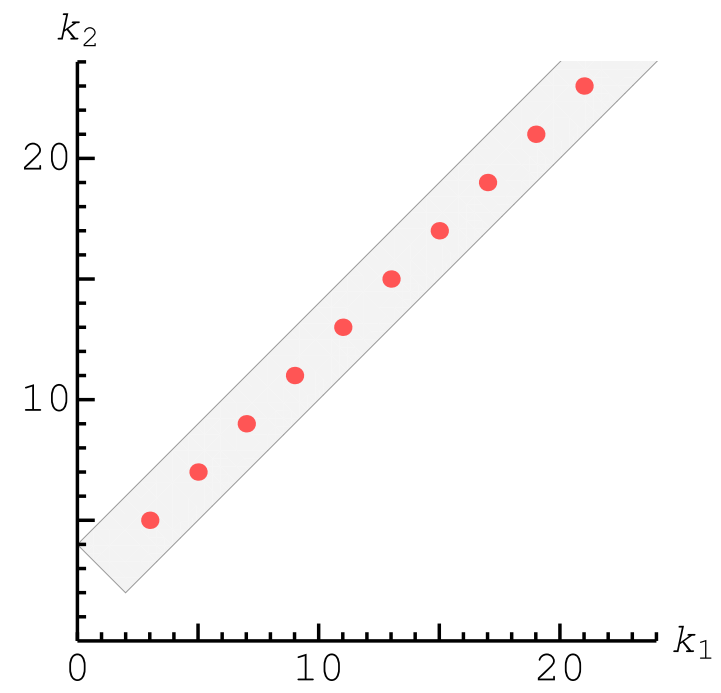

FIG. 4: In the case of $p=4, q=3$, the infinite sequence of $\left(k_{1}, k_{2}\right)=\left(\tilde{a}_{2}, a_{2}\right)$ is shown by dots in the $k_{1}-k_{2}$ plane. The shaded region represents the area that satisfies the inequality (29).

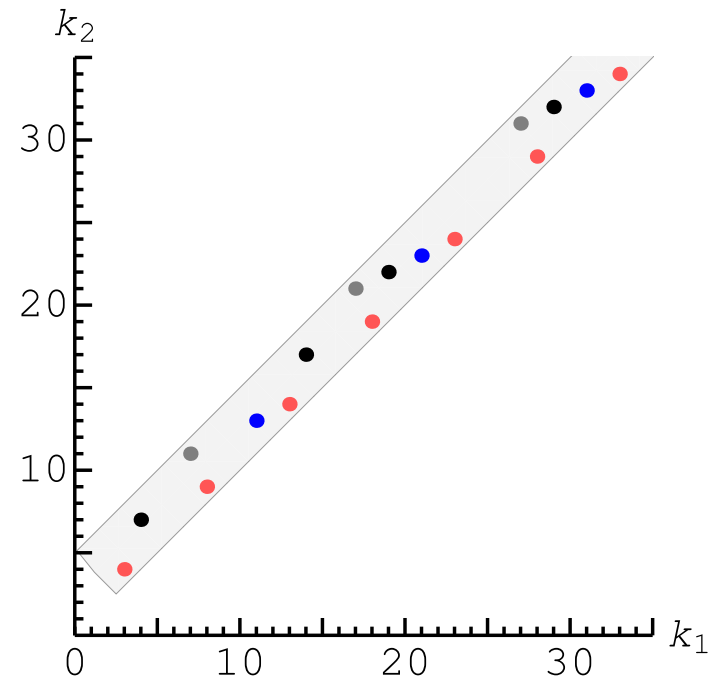

FIG. 5: In the case of $p=5, q=2$, the infinite sequences of $\left(k_{1}, k_{2}\right)=\left(\tilde{a}_{l}, a_{l}\right),(l=1,2,3,4)$ are shown by dots on the line $k_{2}-k_{1}=l$ in the $k_{1}-k_{2}$ plane, respectively.

effectively four-dimensional spacetime near the infinity, and behaves as fully five-dimensional black holes near the horizons. Two black holes are located at the poles on the bolt. Each black hole has an analytic Killing horizon, then there is no curvature singularity on and outside the black hole horizons. We note that although multi-black hole solutions in higher dimensions tend to have non-smooth event horizons [30 36], our solutions have smooth event horizons like multi-black holes with non-trivial asymptotic structure [37]. 
To avoid conical singularities, the spacetime should be quantized by three relatively prime natural numbers $\left(p, p_{+}, p_{-}\right)$satisfying the inequality $0<p_{+}-p_{-}<p<p_{+}+p_{-}$. Then the topology of spatial infinity and that of each black hole are not $S^{3}$, but different lens spaces. The topology of spatial infinity is $L(p ; q)$, while the topologies of the northern and the southern black holes are $L\left(p_{+} ; q_{+}\right)$and $L\left(p_{-} ; q_{-}\right)$, respectively, where $(p, q),\left(p_{+}, q_{+}\right)$, and $\left(p_{-}, q_{-}\right)$are three sets of coprime natural numbers. Then both the topologies and the geometries of the spatial infinity and black holes are uniquely determined by $\left(p, p_{+}, p_{-}\right)$.

On the other hand, when the topology of spatial infinity is fixed as $L(p ; q)$, there exist infinite sequences of coprime natural numbers $\left(p_{+}, p_{-}\right)$that give the same $q$. Using such sequences, we have infinite possibilities of lens space topologies of the northern and the southern black holes $L\left(p_{ \pm} ; q_{ \pm}\right)$for the fixed $(p, q)$. From equation (22), we see that $\left(\sigma_{1}, \sigma_{2}\right)$, equivalently $\left(p_{+}, p_{-}\right)$, can take a variety of values if and only if both parameters $\alpha$ and $\nu$ do not vanish, otherwise, it should hold that $p_{+}=p_{-}$. Therefore, we can obtain the infinite number of possible lens space topologies of black holes, which are determined by $\left(p_{+}, p_{-}\right)$, in the case of Kerr-Taub-bolt base space. The present spacetime is regular on and outside the black hole horizons, and represents a pair of Kaluza-Klein black holes with the rich variety of lens space topologies of horizons, even if the topology of spatial infinity is fixed. We would explain the reason why the solution allows infinite possibilities of horizon topologies under a fixed topology of infinity in the framework of cobordism. However it is still an open question.

The solution (2) can be generalized to a solution of the system including a positive cosmological constant term $-4 \Lambda$ within the integral of the action (11) by replacing the harmonic function (6) into ${ }^{9}$

$$
H(t, r, \theta)=\sqrt{\frac{4 \Lambda}{3}} t+\frac{m_{+}}{r-\mu-\left(r_{b}-\mu\right) \cos \theta}+\frac{m_{-}}{r-\mu+\left(r_{b}-\mu\right) \cos \theta} .
$$

Similar to the cosmological solutions [21, 22], we expect that the metric (21) with the harmonic function (73) describes the physical process such that two black holes with the horizon topologies of $L\left(p_{+} ; q_{+}\right)$and $L\left(p_{-} ; q_{-}\right)$coalesce into a single black hole with the horizon topology of $L(p ; q)$. It would be interesting to compare the coalescence process between

9 Introducing the new coordinate $t^{\prime}=t-\sqrt{3 / 4 \Lambda}$ then taking the limit $\Lambda \rightarrow 0$, the metric (2) with the harmonic function (73) represents the static solution (2) with the harmonic function (6). 
black holes on Kerr-Taub-bolt space and Gibbons-Hawking space [21, 22]. ${ }^{10}$ We leave the analysis for the future.

\section{Acknowledgments}

We would like to thank Benson Way and Yukinori Yasui for the useful discussions. This work is supported by the Grants-in-Aid for Scientific Research No. 19540305 and No. 24540282. MK is supported by a grant for research abroad from JSPS.

\section{Appendix A: Lens space}

The metric of a unit $\mathrm{S}^{3}$ is given by

$$
d s^{2}=d \Theta^{2}+\sin ^{2} \Theta d \Phi^{2}+\cos ^{2} \Theta d \Psi^{2}
$$

with the coordinate range $0 \leq \Theta \leq \pi / 2$ and the identifications

$$
(\Phi, \Psi) \sim(\Phi+2 \pi, \Psi), \quad(\Phi, \Psi) \sim(\Phi, \Psi+2 \pi)
$$

A lens space $L(p ; q)$ is a manifold defined as the quotient of $S^{3}$ under the identification

$$
(\Phi, \Psi) \sim\left(\Phi+2 \pi \frac{q}{p}, \Psi+2 \pi \frac{1}{p}\right)
$$

where $p$ and $q$ are coprime natural numbers. ${ }^{11}$ The lens space $L(p ; q)$ becomes $\mathrm{S}^{3}$ in the case of $p=1$, but it has a different topology from $S^{3}$ if $p \neq 1$. It is known that two lens spaces $L(p ; q)$ and $L\left(p^{\prime} ; q^{\prime}\right)$ are diffeomorphic if and only if the following two conditions are satisfied: (i) $p=p^{\prime}$, (ii) $\left(q \pm q^{\prime} \bmod p\right)=0$ or $\left(q q^{\prime} \pm 1 \bmod p\right)=0$. For this reason, it is sufficient to consider the case $p>q$. Fig 6 represents the identifications for $L(p ; q)$.

Note that identical points same as the origin $(\Phi, \Psi)=(0,0)$ induced by the identifications (A2) and (A3) are described as

$$
(\Phi, \Psi)=\left(2 \pi\left(\frac{q}{p} a-b\right), 2 \pi \frac{a}{p}\right),
$$

$\overline{10}$ The solutions [21, 22] describe the coalescence of two black holes whose topologies are $L\left(p_{1} ; 1\right)$ and $L\left(p_{2} ; 1\right)$ into a single black hole with the topology of $L\left(p_{1}+p_{2} ; 1\right)[38,39]$.

11 The case of $p<0$ or $q<0$ can be considered as a matter of the definition of signs of coordinates $\Phi$ or $\Psi$. Note that $q$ can also take 0 only in the case of $p=1$. 


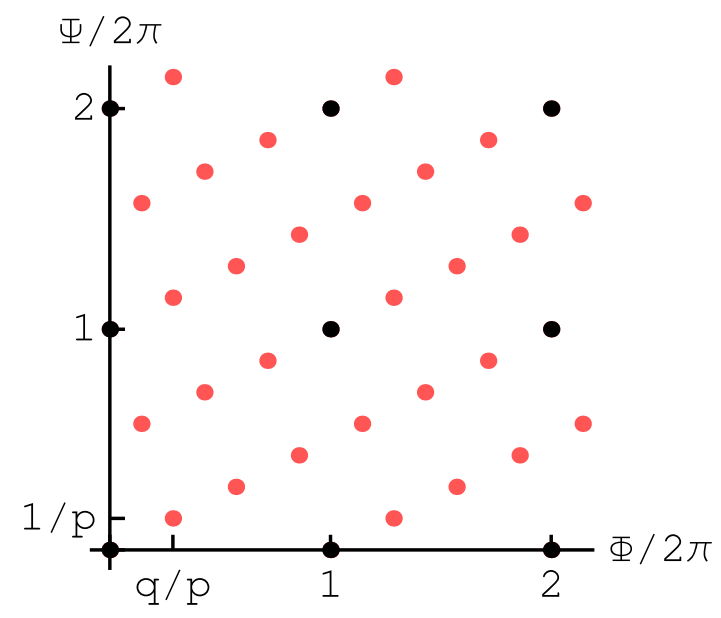

FIG. 6: The identifications for $L(p ; q)$. As an example we depicted the identical points same as $(\Phi, \Psi)=(0,0)$ for $L(7 ; 2)$. The black and red points come from the identifications (A2) and (A3), respectively. The number of the points in the region $0<\Phi / 2 \pi<1$ and $0<\Psi / 2 \pi<1$ is $p-1$, and the number of possible lines with gradient $1 / q$ through at least one of the points in this region is $q$.

where $a$ and $b$ are integers. If $a$ and $b$ are coprime natural numbers, there is no identical point same as the origin along the finite line connected with the origin and the point (A4). The area of a unit lens space $L(p ; q)$ whose local metric is given by (A1) becomes

$$
\mathcal{A}_{L(p ; q)}=2 \pi^{2} / p
$$

If we use Euler coordinates $(\theta, \phi, \psi)$ defined by

$$
\Theta=\frac{\theta}{2}, \quad \Phi=\frac{\psi-\phi}{2}, \quad \Psi=\frac{\psi+\phi}{2},
$$

the metric (A1) becomes

$$
d s^{2}=\frac{1}{4}\left[d \theta^{2}+\sin ^{2} \theta d \phi^{2}+(d \psi+\cos \theta d \phi)^{2}\right] .
$$

In these coordinates, $0 \leq \theta \leq \pi$, and the identifications (A2) and (A3) become

$$
\begin{aligned}
& (\phi, \psi) \sim(\phi \pm 2 \pi, \psi+2 \pi) \\
& (\phi, \psi) \sim(\phi+2 \pi(1-q) / p, \psi+2 \pi(1+q) / p) .
\end{aligned}
$$

\section{Appendix B: Regularity condition on the bolt}

In this section, we study the regularity condition on the bolt off the black hole, i.e., $r=r_{b}$ and $\theta \neq 0, \pi$. The metric of four-dimensional Kerr-Taub-bolt space (4) diverges 
at $r=r_{b}$ apparently. These points correspond to fixed points of the Killing vector field $\partial / \partial \psi+2 \alpha \nu /\left(r_{b}^{2}-\nu^{2}-\alpha^{2}\right) \partial / \partial \phi$. We expand the metric (2) near these fixed points, and obtain regularity conditions of identifications.

Introducing new coordinates,

$$
R=\sqrt{\frac{2\left(r-r_{b}\right)}{r_{b}-\mu}}, \quad \chi=\frac{-2 \alpha \nu}{r_{b}^{2}-\nu^{2}-\alpha^{2}} \psi+\phi,
$$

near $r=r_{b}$, the metric (2) behaves as

$$
\begin{aligned}
d s^{2} \simeq-H\left(r_{b}, \theta\right)^{-2} d t^{2}+ & H\left(r_{b}, \theta\right) \Xi\left(r_{b}, \theta\right)\left[d R^{2}+R^{2}\left(\frac{r_{b}-\mu}{\Xi\left(r_{b}, \theta\right)}\left(2 \nu \cos \theta+\alpha \sin ^{2} \theta\right) d \chi\right.\right. \\
& \left.\left.+\frac{2 \nu\left(r_{b}-\mu\right)}{r_{b}^{2}-\nu^{2}-\alpha^{2}} d \psi\right)^{2}+d \theta^{2}+\frac{\left(r_{b}^{2}-\nu^{2}-\alpha^{2}\right)^{2}}{\Xi\left(r_{b}, \theta\right)^{2}} \sin ^{2} \theta d \chi^{2}\right] .
\end{aligned}
$$

The induced metric on the two-dimensional bolt is given by the second and the third terms in the second line of the above metric. The function $\Xi\left(r_{b}, \theta\right)=r_{b}^{2}-(\nu-\alpha \cos \theta)^{2}$ takes larger value in the north part than the south part, so the shape of the bolt is like a pear. In fact, this asymmetric behavior leads a difference of topologies of each black holes.

Near the bolt $R=0$, the metric (B) with $t, \theta, \chi=$ const. takes the form

$$
\left.d s^{2}\right|_{t, \theta, \chi=\text { const. }} \simeq H\left(r_{b}, \theta\right) \Xi\left(r_{b}, \theta\right)\left[d R^{2}+R^{2}\left(\frac{2 \nu\left(r_{b}-\mu\right)}{r_{b}^{2}-\nu^{2}-\alpha^{2}} d \psi\right)^{2}\right] .
$$

The above two-dimensional metric is regular if and only if the coordinate $\psi$ is periodic with a period $2 \pi\left(r_{b}^{2}-\nu^{2}-\alpha^{2}\right) /\left(2 \nu\left(r_{b}-\mu\right)\right)$ along $\chi=-2 \alpha \nu \psi /\left(r_{b}^{2}-\nu^{2}-\alpha^{2}\right)+\phi=$ const. in $(\phi, \psi)$ plane. Equivalently, such the condition is same as the following identification

$$
(\phi, \psi) \sim\left(\phi+2 \pi \frac{\alpha}{r_{b}-\mu}, \psi+\pi \frac{r_{b}^{2}-\nu^{2}-\alpha^{2}}{\nu\left(r_{b}-\mu\right)}\right) .
$$

Using the coordinates (19), we see that the regularity condition (B4) coincides with the condition (25).

[1] P. Dobiasch and D. Maison, Gen. Rel. Grav. 14, 231 (1982).

[2] G. W. Gibbons and D. L. Wiltshire, Annals Phys. 167, 201 (1986) [Erratum-ibid. 176, 393 (1987)].

[3] J. P. Gauntlett, J. B. Gutowski, C. M. Hull, S. Pakis and H. S. Reall, Class. Quant. Grav. 20, 4587 (2003) arXiv:hep-th/0209114. 
[4] D. Gaiotto, A. Strominger, X. Yin, JHEP 0602, 024 (2006). hep-th/0503217.

[5] H. Ishihara and K. Matsuno, Prog. Theor. Phys. 116, 417 (2006) arXiv:hep-th/0510094.

[6] H. Ishihara, M. Kimura, K. Matsuno and S. Tomizawa, Class. Quant. Grav. 23, 6919 (2006) [arXiv:hep-th/0605030].

[7] K. Matsuno, H. Ishihara, T. Nakagawa and S. Tomizawa, Phys. Rev. D 78, 064016 (2008) arXiv:0806.3316 [hep-th]].

[8] Y. Chen and E. Teo, Nucl. Phys. B 850, 253 (2011) [arXiv:1011.6464 [hep-th]].

[9] P. G. Nedkova and S. S. Yazadjiev, Phys. Rev. D 84, 124040 (2011) [arXiv:1109.2838 [hep-th]].

[10] T. Tatsuoka, H. Ishihara, M. Kimura and K. Matsuno, Phys. Rev. D 85, 044006 (2012) arXiv:1110.6731 [hep-th]].

[11] K. Matsuno, H. Ishihara, M. Kimura and T. Tatsuoka, Phys. Rev. D 86, 104054 (2012) arXiv:1208.5536 [hep-th]].

[12] S. Tomizawa and S. 'y. Mizoguchi, Phys. Rev. D 87, 024027 (2013) arXiv:1210.6723 [hep-th]].

[13] C. Stelea, C. Dariescu and M. A. Dariescu, Phys. Rev. D 87, 024039 (2013) arXiv:1211.3154 $[\mathrm{gr}-\mathrm{qc}]]$.

[14] Y. Kanou, H. Ishihara, M. Kimura, K. Matsuno and T. Tatsuoka, Phys. Rev. D 90, 084004 (2014) arXiv:1408.2956 [hep-th]].

[15] S. Tomizawa and H. Ishihara, Prog. Theor. Phys. Suppl. 189, 7 (2011) arXiv:1104.1468 [hepth]].

[16] S. D. Majumdar, Phys. Rev. 72, 390 (1947).

[17] A. Papapetrou, Proc. R. Ir. Acad. Sect. A 51, 191 (1947).

[18] R. C. Myers, Phys. Rev. D 35, 455 (1987).

[19] J. P. Gauntlett, R. C. Myers and P. K. Townsend, Class. Quant. Grav. 16, 1 (1999) hep-th/9810204.

[20] H. Ishihara, M. Kimura, K. Matsuno and S. Tomizawa, Phys. Rev. D 74, 047501 (2006) hep-th/0607035.

[21] H. Ishihara, M. Kimura and S. Tomizawa, Class. Quant. Grav. 23, L89 (2006) hep-th/0609165.

[22] K. Matsuno, H. Ishihara, M. Kimura and S. Tomizawa, Phys. Rev. D 76, 104037 (2007) arXiv:0707.1757 [hep-th]].

[23] K. -i. Maeda, N. Ohta, M. Tanabe, Phys. Rev. D74, 104002 (2006). hep-th/0607084. 
[24] G. W. Gibbons and M. J. Perry, Phys. Rev. D 22, 313 (1980).

[25] J. Kunz and S. Yazadjiev, Phys. Rev. D 79, 024010 (2009) [arXiv:0811.0730 [hep-th]].

[26] J. Kunz, P. G. Nedkova and C. Stelea, Nucl. Phys. B 874, 773 (2013) [arXiv:1304.7020 [gr-qc]].

[27] A. M. Ghezelbash, R. B. Mann and R. D. Sorkin, Nucl. Phys. B 775, 95 (2007) hep-th/0703030.

[28] Y. Chen and E. Teo, Nucl. Phys. B 838, 207 (2010) [arXiv:1004.2750 [gr-qc]].

[29] H. K. Kunduri and J. Lucietti, Phys. Rev. Lett. 113, 211101 (2014) arXiv:1408.6083 [hep-th]].

[30] G. W. Gibbons, G. T. Horowitz and P. K. Townsend, Class. Quant. Grav. 12, 297 (1995) hep-th/9410073.

[31] D. L. Welch, Phys. Rev. D 52, 985 (1995) arXiv:hep-th/9502146].

[32] G. N. Candlish and H. S. Reall, Class. Quant. Grav. 24, 6025 (2007) [arXiv:0707.4420 [gr-qc]].

[33] G. N. Candlish, Class. Quant. Grav. 27, 065005 (2010) [arXiv:0904.3885 [hep-th]].

[34] C. N. Gowdigere, A. Kumar, H. Raj and Y. K. Srivastava, arXiv:1401.5189 [hep-th].

[35] C. N. Gowdigere, arXiv:1407.5338 [hep-th].

[36] M. Kimura, H. Ishihara, K. Matsuno and T. Tanaka, Class. Quant. Grav. 32, 015005 (2015) arXiv:1407.6224 [gr-qc]].

[37] M. Kimura, Phys. Rev. D 78, 047504 (2008) arXiv:0805.1125 [gr-qc]].

[38] C. M. Yoo, H. Ishihara, M. Kimura, K. Matsuno and S. Tomizawa, Class. Quant. Grav. 25, 095017 (2008) arXiv:0708.0708 [gr-qc]].

[39] M. Kimura, H. Ishihara, S. Tomizawa and C. M. Yoo, Phys. Rev. D 80, 064030 (2009) arXiv:0906.4681 [gr-qc]]. 